\title{
Research on Routing Protocol of Mobile Ad Hoc Network
}

\author{
Ming Fu TUO, Min CHE
}

Air Force Engineering University,Xi'An, China

\begin{abstract}
Routing protocol is an important content of mobile Ad Hoc. The typical mobile Ad Hoc network routing protocols is introduced. The advantages and disadvantages of these routing protocols are analyzed. A hybrid routing protocol is put forward based on this.
\end{abstract}

\section{Introduction}

Mobile Ad Hoc network is usually composed of a set of wireless mobile nodes. It doesn't depend on existing fixed communication network infrastructure. It does not have any central entity. It is a type of self-organization and self-healing network. Nodes in Mobile Ad Hoc network can move quickly and the positions of the network node are rapidly changing. Each node in the network are both routers and hosts. They communicate by sending and receiving data packet[1-3].

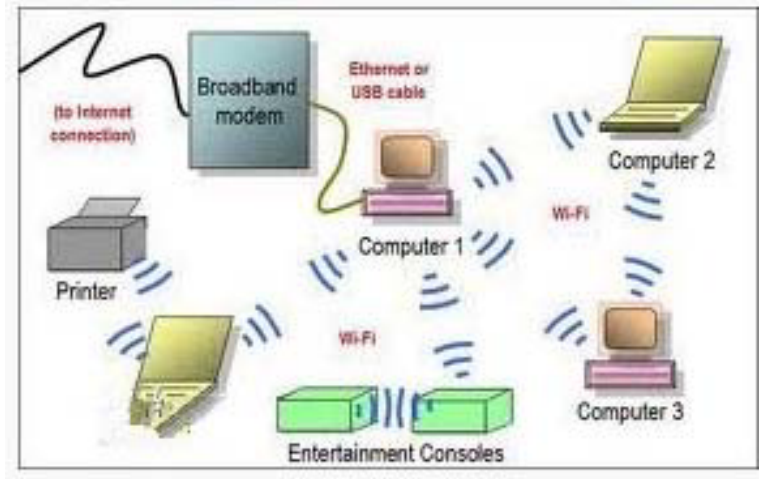

Figure 1. Mobile Ad Hoc network.

\section{The characteristics of mobile Ad Hoc}

The main characteristics of mobile Ad Hoc are as follows:

(1)The network topology structure changes frequently. There is no fixed in Ad Hoc communications facilities and central management equipment, network node can randomly move in any direction at any speed, combined with wireless transmitters sending power change, the influence of the environment and the factors such as mutual interference between signals, can cause the dynamic change of network topology[4].

(2) Limited resources. Energy for mobile host in Ad Hoc working energy is limited. The network performance will degrade as the energy decreases. On the other hand, there limited bandwidth in mobile Ad Hoc network, and the signals may bring interference. The available transmission bandwidth of mobile host will be far less than the maximum theoretical bandwidth.

(3) Multiple hops communications. Two network nodes will not be in the same network because of the limitation of available resources. This problem can be solved by multiple hops between the source host and the destination host[5].

(4) Low security. Ad Hoc inter-nodes communicates by the wireless channel. The transmission of information is very vulnerable to listen and retransmission, tampered with, forge attack, if a routing protocol once suffered the malicious attacks, the self-organizing networks would be unable to work normally[6].

\section{Unicast routing protocol}

There are three communication ways in Ad hoc networks, that is, unicast, multicast broadcast. 
The mobile Ad Hoc network routing protocols can be divided into table driven routing protocol and on-demand routing protocols according to the routing driven mode.

Table driven routing protocol, also called a priori type routing protocols, each node in the network to maintain one or more tables to store other nodes in the routing information, through radio and update information to maintain routing tables and consistency between the change of network topology. Once the source node to send a message, can be gained through the look-up table immediately arrived at the destination node routing. Typical table driven routing protocol includes: DSDV (destination - sequenced short - vector routing), CGSR (cluster head gateway switch r outing) and WRP (wireless routing protocol), etc.

On-demand routing protocols, also called reactive routing protocols, it is a kind of when need to send the data to find the routing routing algorithm. In this kind of routing protocol, the node does not need to maintain the routing information accurately and in time, when the destination node to send it a message, only the source node in the network routing lookup process, find the corresponding routing. Compared with the prior type routing protocols, reactive routing protocol overhead is small, but the datagram transmission delay is larger. Commonly used reactive routing protocol AODV (Adhoc on - demand vector routing), DSR (dynamic source routing), TORA (temporally ordered routing algorithm), etc.

Logic structure according to the network, it can be divided into the flat structure routing protocols and hierarchical structure of two kinds of routing protocols.

In the flat structure routing protocols, each communication nodes are equal. Common agreement include DSDV, WRP, FSR and AODV, and so on. When network scale, the flat structure routing protocol performance decline rapidly.

The more widespread use of hierarchical Routing protocols are mainly CGSR (Cluster head Gateway Switch Routing), ZRP and LEACH agreement. CGSR routing protocol is based on DSDV prior type, in CGSR network model, the network was divided into overlapping groups, each group by group of first selection algorithm to select a group first. Each node holds a group of members of the table and routing table, the former record of each node in the network group of the first and periodic broadcast updates; The latter group for each save a table item, record the next node to the group first. In two or more group of the first communication within the scope of the node as a gateway node, group of the first must be through the gateway to realize.

ZRP protocol use based on ideas, but do not produce group of the first group of use should be first type routing protocols, group of on-demand routing between the arms. LEACH agreement also use group and gateway to mutual information, and use dynamic way to group of the first election

All three of these protocols has itself advantages and disadvantages, CGSR protocol response speed, but expensive. ZRP combines prior type and the advantages of on-demand routing, but slow route setup between group, and establish a route using flood, large energy consumption. LEACH agreement through the rotation group of the first random election, balanced relay communication business, but the node with the same probability in the agreement first competition group, factors such as the lack of energy, and must ensure that all nodes are able to direct communication with a gateway node. Another CGSR and LEACH group information must be used between the group first and gateway transmission, increased the group of the first, and the gateway load at the same time, the routing is not necessarily optimal.

\section{Broadcast routing protocol}

\subsection{Reverse path forwarding broadcast protocol}

Reverse path forwarding a bit is both efficiency and simplicity. Reverse path forwarding agreement, node after receiving the broadcast packets to check whether the packet is the latest package had never received. If so, that the package is the path to the source to the shortest path of the node, the node will receive a packet to all nodes except to forward; Otherwise don't do any processing, discards the packet. Agreement on the basis of was the first to arrive packets often corresponds to the shortest path. 


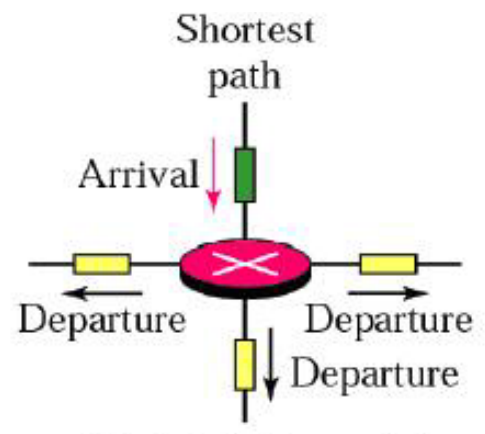

a. Packet is forwarded

Figure 2 Reverse path forwarding broadcast protocol

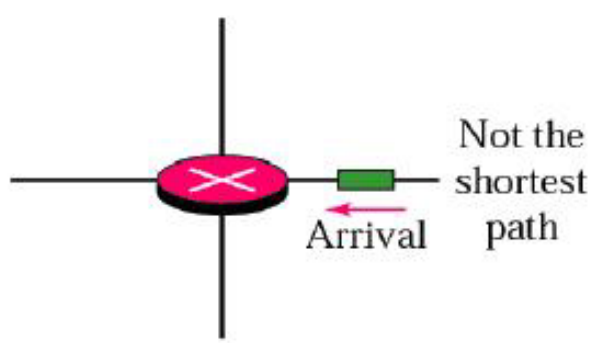

\section{b. Packet is discarded}

Figure 3 Reverse path forwarding broadcast protocol b

\subsection{Support the tree broadcast protocol}

Support all node tree agreement save a spanning tree, it is a subset of the subnet, it contains all of the nodes, but does not contain any ring. Every node knows to link its which lines belong to the spanning tree. When a packet arrived at nodes, it will forward it to all other spanning tree except arrival path path, this method can save the bandwidth, and generates a copy of the group is the least. The downside to this method is that each node must save a spanning tree.

\section{Conclusions}

Mobile Ad Hoc network has self-organizability, multiple hops and characteristics on the dynamic change of network topology, the special of the network make the traditional cable network technology can not be directly used in Ad Hoc network, which requires for Ad Hoc network many new protocols and technologies, such as channel access technology, security technology, routing technology, etc. This article mainly focus on the in $\mathrm{Ad}$ Hoc network routing technology.

\section{References}

1.Sung-Ju Lee,Matio Geria. Split Multipath Routing with Maximally Disjoint Paths in Ad Hoc Networks. IEEE 2011,10 (6):3201-3205.

2.Mahesh K.Marina, Samir R.Das.On-demand Multipath Distance Vector Routing in Ad Hoc Networks. Network Protocols,2011.Ninth International Conference. 2001.

3.Stephen Mueller, Rose P .Tsang, Dipak Ghosal. Multipath Routing in Mobile Ad Hoc N etworks:Issues andC hallenges.2014,

4.Prasant Mohapatra, Jian Li, ChaoG ui." QoSin M obileA dH ocN etworks".2012,

5.Mingfu Tuo,Xingshe Zhou.etc.A method for Cyber-Physical System behavior modeling and safety verification based on extended hybrid system description language,ICEICE 2016.

6.Das S.K.,Mukherjee A , BandyopadhyayS , PaulK ." Improving Quality-of-Service in Ad Hoc Wireless Networks with Adaptive Multi-path Routing".IEEE Global Telecommunications Conference. 2015 . 